\title{
Far Wing Asymmetry of Rotational Raman Lines in Hydrogen
}

\author{
Magnus Gustafsson \\ Department of Chemistry, University of Gothenburg, SE 41296 Gothenburg, Sweden \\ Correspondence should be addressed to Magnus Gustafsson, magngu@chem.gu.se
}

Received 22 April 2009; Accepted 8 July 2009

Academic Editor: Chantal Stehle

Copyright () 2010 Magnus Gustafsson. This is an open access article distributed under the Creative Commons Attribution License, which permits unrestricted use, distribution, and reproduction in any medium, provided the original work is properly cited.

Depolarized Raman spectra of compressed hydrogen gas have been computed rigorously previously for $36 \mathrm{~K}$ and $50 \mathrm{~K}$ (Gustafsson et al. (2009)). The far wings of the rotational lines show asymmetry that goes beyond that expected from the theory for intracollisional interference and Fano line shapes. Here we analyze the $S_{0}(0)$ line for pure hydrogen at $36 \mathrm{~K}$ in detail. The added asymmetry stems partly from a shape resonance which adds significant intensity to the higher frequency side of the line profile. The influence of the threshold energy for the rotational transition accounts for the remainder.

\section{Introduction}

The depolarized Raman spectrum of hydrogen shows a number of spectral lines corresponding to rotational transitions with $\Delta j_{n}=0, \pm 2$ in hydrogen molecule number $n$ [1]. These spectral features are broadened due to collisions between the molecules and the corresponding width is approximately inversely proportional to the time between the collisions. The transitions with $\Delta j_{n}=2$ give rise to the so-called $S$ lines which are located on the Stokes side of the spectrum where the energy of the scattered photon is lower than that of the incident photon. The incident light may also scatter from pairs of hydrogen molecules in an interaction-induced Raman process $[2,3]$. The pairs are transient complexes with a lifetime corresponding to the duration of the $\mathrm{H}_{2}-\mathrm{H}_{2}$ collisions. In gases under pressures of less than a few hundred atmospheres the time between collisions is typically much longer than the duration of the collisions. This implies that the spectral features due to interaction-induced light scattering are much broader than the pressure-broadened $S$ lines.

At high gas pressures, collisionally interacting triples of molecules will also scatter the light. In general the Raman intensity may be expressed as a virial expansion [4] in powers of the gas density, $\rho$, with the terms proportional to $\rho, \rho^{2}, \rho^{3}$, and so forth, where the second and third term correspond to binary and ternary collisions, respectively. The terms which is linear in the density will come into play if broadening mechanisms other than collisions dominate, such as Doppler or natural line broadening. Here, the temperature is low enough that Doppler broadening can be ignored as well as the natural line width which is extremely small for rotational states. Furthermore, in this work I consider the far wings of the $S$-lines with frequency shifts on the order of $20 \mathrm{~cm}^{-1}$ from the line centers. Small detunings, that is, frequencies approaching the line center, imply that a higher number of subsequent collisions have to be taken into account, and a higher number of terms in the virial expansion have to be included. Detunings of about $20 \mathrm{~cm}^{-1}$ are large enough so that single binary $\left(\mathrm{H}_{2}-\mathrm{H}_{2}\right)$ collisions alone contribute to the Raman intensity. The density-squared component of the intensity is also what has been extracted in the experiments that are relevant for this work [5].

The impact approximation [6, 7] for collisional broadening considers single or several subsequent collisions and can thus describe spectral lines at arbitrarily small detuning. It does not, however, fully include the effects from mixing of molecular states, such as the $\mathrm{H}_{2}$ rotational levels, $j_{n}$. The spectra in this work are computed with a close-coupling scheme $[8,9]$ for the diatom-diatom scattering including the angular momentum couplings exactly [10]. The method is an extension of the radiative close-coupling theory [11] and it is valid far from the line centers and for asymptotically forbidden transitions. Thus it provides a correct description of the wings of the monomer-allowed spectral lines, where only isolated two-body collisions need to be considered. 


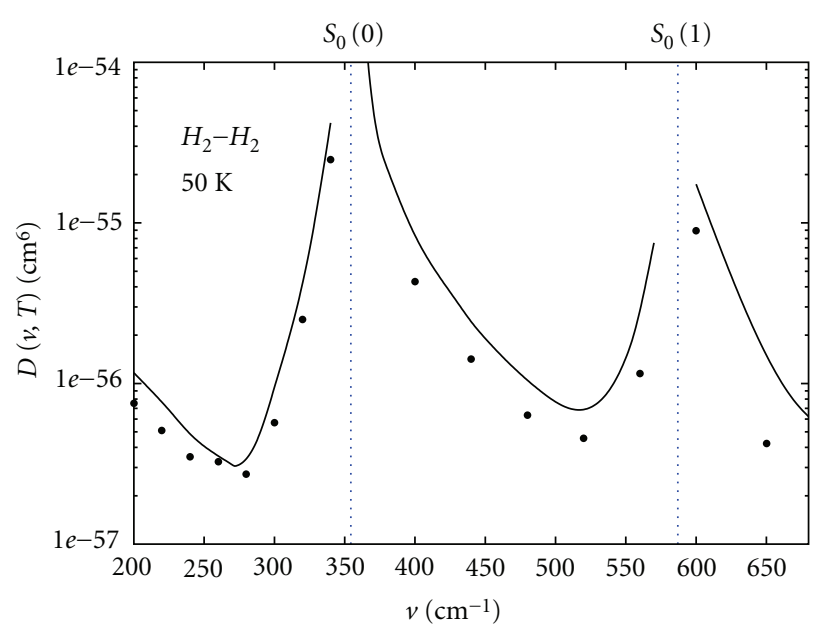

Figure 1: The $S_{0}(0)$ and $S_{0}(1)$ lines of the depolarized Raman spectrum of hydrogen $\left(65 \%\right.$ para- $\mathrm{H}_{2}, 35 \%$ ortho- $\left.\mathrm{H}_{2}\right)$ at $50 \mathrm{~K}$. The frequency of the rotational transitions are indicated with vertical blue dotted lines. The solid curves represent the computed data [10] and the filled circles a measurement [5].

Theory of far wings has been reviewed recently in chapter $\mathrm{V}$ of the book by Hartmann et al. [12]. A perturbative theoretical treatment which includes intracollisional interference was developed by [13] and applied to $\mathrm{H}_{2}-\mathrm{H}_{2}$ light scattering. Furthermore, a nonperturbative model based on the sudden description of the collisions $[14,15]$ has been applied to the depolarized Raman spectrum of nitrogen. The calculation in [10], which produced the theoretical data analysed in this work, does not rely on either of those approximations. It is an exact description of the collisional dynamics when vibrational and higher energy modes may be ignored and it represents the most accurate computation of the densitysquared component of the hydrogen Raman spectrum.

\section{Computed Spectra}

The details of the calculation of the depolarized light scattering in compressed hydrogen gas may be found in [10]. Single binary collisions were taken into account, allowing for evaluation of the virial term of the Raman cross section which is proportional to the density squared. The closecoupling scattering calculations were carried out on the potential surface by Schäfer and Köhler [16] using the static collision-induced polarizability surface from [17]. It should be noted that the latter differs from the true dynamic polarizability by an amount which is thought to be several percent $[2,3]$.

The depolarized Raman intensity at a fixed temperature is evaluated through

$$
D(\nu, T)=\lambda_{0}^{3} \sum_{j_{1 i} j_{2 i}} P_{j_{1 i}} P_{j_{2 i}} \int_{0}^{E_{\max }} e^{-E / k T} D_{j_{1 i} j_{2 i}}(\nu, E) d E
$$

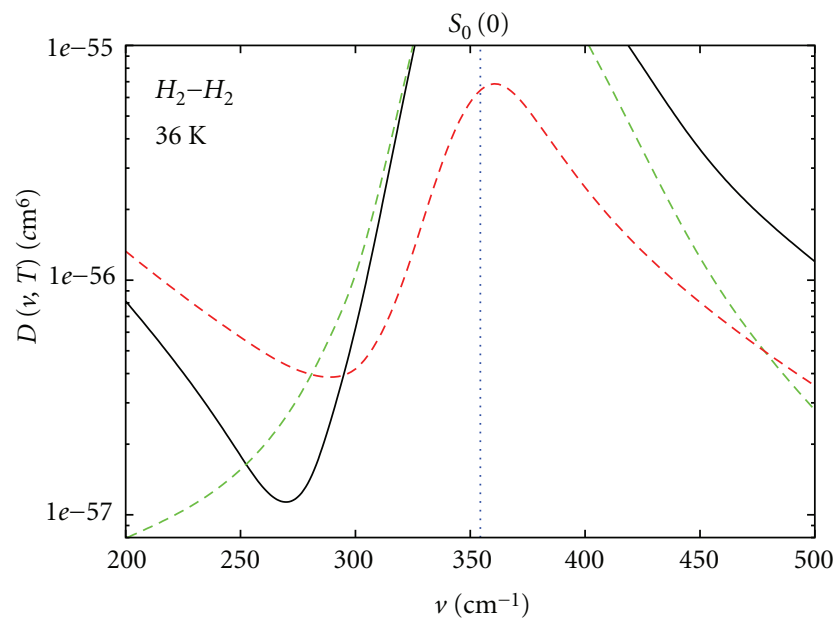

Figure 2: The $S_{0}(0)$ line of the depolarized Raman spectrum of pure hydrogen at $36 \mathrm{~K}$. The frequency of the rotational transition is indicated with the vertical blue dotted line. The solid curve represents the computed data [10]. Computed data where only the permanent polarizability is included is indicated with a green dashed curve. Similarly, the spectrum due only to collision-induced polarizability is indicated with a red dashed curve.

with the energy-dependent intensity defined by

$$
D_{j_{1 i} j_{2 i}}(\nu, E)=\frac{2 c v_{s}^{2}}{\phi_{0} \phi_{s} h} \sum_{i^{*}} \sum_{f}\left|S_{i}^{f}(E)\right|^{2},
$$

where the scattering matrix element $S_{i}^{f}(E)$ for initial and final angular momentum states $i$ and $f$, corresponding to incident and scattered photons with frequencies $\nu_{0}$ and $v_{s}$, respectively. $\lambda_{0}$ is the thermal de Broglie wave length, $\phi_{0}$ and $\phi_{s}$ are the incident and scattered photon fluxes, respectively, in units $\mathrm{s}^{-1} \mathrm{~cm}^{-2}$ and $P_{j}(T)$ is the rotational population for $\mathrm{H}_{2}$ at temperature $T$. The summation over $i^{*}$ indicates all initial angular momenta except for $j_{1 i}$ and $j_{2 i}$. It has been verified that an upper integration limit of $E_{\max }=13 \mathrm{kT}$ provides convergence in (1).

Figure 1 shows the Raman spectrum at $50 \mathrm{~K}$ which is dominated by the wings of the $S(0)$ and $S(1)$ lines, except for at $v<270 \mathrm{~cm}^{-2}$ where the purely translational collisioninduced band appears. The frequency shift $\nu=\nu_{0}-$ $v_{s}$ is positive for Stokes scattering. Experimental Raman intensities are also shown and the agreement of the line shapes is satisfactory. Considerable difficulty in the ab initio calculations of the potential and polarizability surfaces should be taken into account. Due to the low temperature only two $S$ lines appear in the spectrum; all the paraand ortho-hydrogen molecules are in their lowest rotational states 0 and 1 , respectively.

In Figure 2 the theoretical result for pure parahydrogen at $36 \mathrm{~K}$ is presented. The results when permanent and collisioninduced polarizabilities (one at the time) are artificially turned off are also displayed to aid the analysis of the asymmetric broadening. There is strong destructive interference between the permanent and collision-induced components on the low frequency side of the $S_{0}(0)$ transition; inclusion 


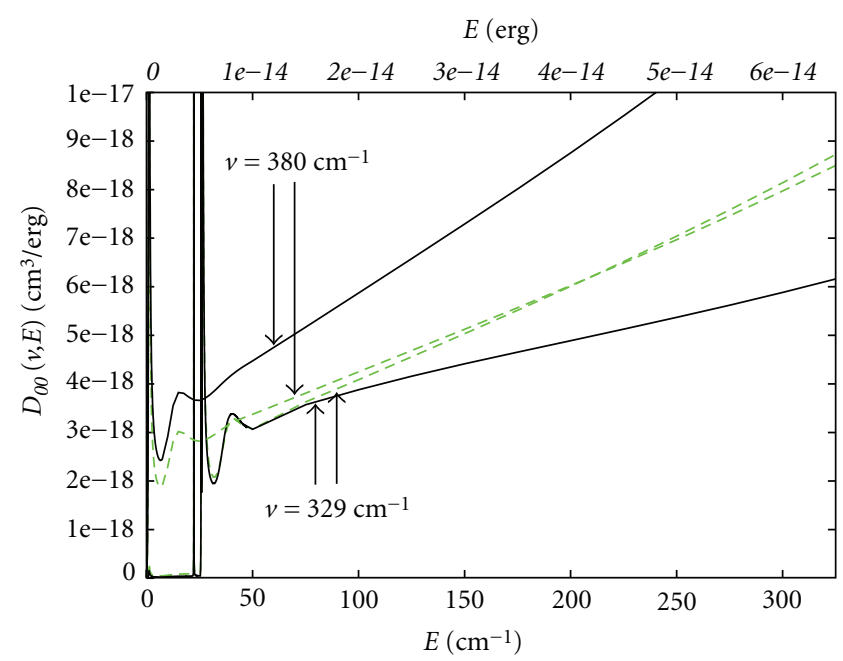

FIGURE 3: The light scattering intensity versus collision energy for frequency shifts of $329 \mathrm{~cm}^{-1}$ and $380 \mathrm{~cm}^{-1}$. The result where the collision-induced part is excluded is shown with green dashed lines.

of both components gives a lower intensity than each of them alone around $280 \mathrm{~cm}^{-1}$. The high frequency side, on the other hand, shows an equally strong constructive interference.

\section{Rotational Line Shapes}

It is helpful for the line shape analysis to briefly review the absorption spectroscopy analogue to the process at hand. The HD molecule has a tiny permanent dipole moment [18] and its infrared spectrum has been investigated in great detail both experimentally $[19,20]$ and theoretically $[21-23]$. For example, in the spectrum of HD molecules in a helium bath it has been established that an interference of the HD dipole with the interaction-induced HD-He dipole gives rise-to-socalled Fano line profiles for the $R$ and $P$ transitions. The low density limit of the Fano line shape has a symmetric Lorentzian term and an antisymmetric interference term [24]. It appears that the mechanism should be the same in a Raman spectrum when a permanent and an interactioninduced polarizability interfere.

In the pioneering theoretical work on the depolarized Raman line shapes in hydrogen Borysov and Moraldi [13, 25] assumed a three component contribution to the intensity: a pressure broadened allowed line described by a Lorentzian, a collision-induced quasicontinuum, and the interference of those two. For the first of those contributions the impact approximation predicts a pressure-broadened symmetric line shape described by a Lorentzian. If the collisioninduced polarizability is removed from the calculation of the spectrum such a line shape is expected. The dashed (green) curve in Figure 2 shows our theoretical result for the allowed $S$-line which is clearly not symmetric. Intracollisional interference accounts for some of the added asymmetry when the collision-induced polarizability is included in the calculation, that is, when the solid black curve deviates from

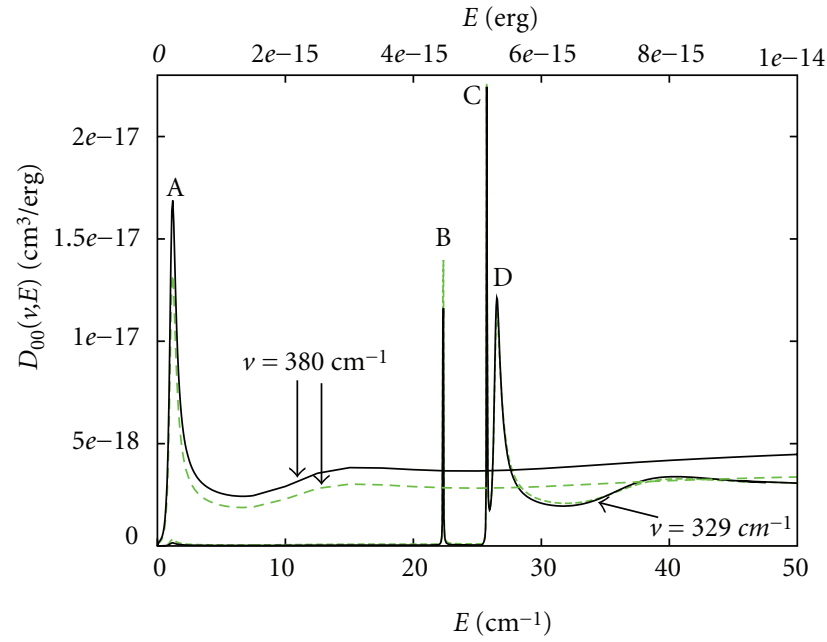

FIgURE 4: Same as in Figure 3 but for a smaller energy range. For $380 \mathrm{~cm}^{-1}$ there is a shape resonance due to $\left(j_{1}, j_{2}, l, J\right)=(0,0,2,2)$ which is marked with an A. For $329 \mathrm{~cm}^{-1}$ there is structure due to a predissociating state $\left(j_{1}, j_{2}, l, J\right)=(0,2,0,2)$ (B) and shape resonances on the $\left(j_{1}, j_{2}\right)=(0,2)$ potential $(\mathrm{C}$ and $\mathrm{D})$.

the sum of the dashed red and green curves. The asymmetry that is observed when only the permanent polarizability is included must, however, come about through a different mechanism.

\section{Analysis of $D_{o o}(v, E)$}

In the following analysis I will consider the case of pure parahydrogen with the spectrum presented in Figure 2. It should be noted that, due to the low temperature, all $\mathrm{H}_{2}$-molecules are initially in their rotational ground state, $j_{n}=0$. The Raman intensity versus collision energy, $D_{00}$, which is given in (2) is shown for two frequency shifts in Figure 3 over the whole energy interval required for an accurate evaluation of the Boltzmann average in (1). The two frequency shifts, 329 and $380 \mathrm{~cm}^{-1}$, were chosen so that they are positioned roughly symmetrically relative to the $S_{0}(0)$ transition at $354.4 \mathrm{~cm}^{-1}$. The allowed contribution to the intensity is shown with dashed green curves and those virtually coincide for 329 and $380 \mathrm{~cm}^{-1}$ above $E$ $50 \mathrm{~cm}^{-1}$. One may thus conclude that it is the low energy behaviour that introduces the asymmetry that goes beyond the intracollisional interference effect, which is displayed as the difference between the solid black curves in Figure 3.

Figure 4 shows the same intensities for a low energy range. For the frequency shift $\nu=380 \mathrm{~cm}^{-1}$ a feature (A) around $E=1.2 \mathrm{~cm}^{-1}$ is now clearly visible. A computation of the cross section for different total angular momenta $J$ (not reported here) shows that the partial wave with $J=l=2$ alone gives rise to the feature. Thus it is consistent with a shape resonance on the $l=2$ effective potential

$$
V_{\text {eff }}(R)=V_{000}(R)+\frac{\hbar^{2} l(l+1)}{2 m R^{2}},
$$


TABLE 1: Raman intensity $D$ at $\nu=380 \mathrm{~cm}^{-1}$ and $T=36 \mathrm{~K}$ evaluated with three different upper energy limits, $E_{\max }$, in the integral of (1).

\begin{tabular}{lcccc}
\hline$E_{\max }[\mathrm{erg}]$ & $E_{\max }\left[\mathrm{cm}^{-1}\right]$ & $E_{\max } / k T$ & $D(\nu, T)\left[\mathrm{cm}^{6}\right]$ & fraction $[\%]$ \\
\hline $9.93 \times 10^{-16}$ & 5 & 0.200 & $0.103 \times 10^{-54}$ & 21.5 \\
$4.965 \times 10^{-15}$ & 25 & 1.00 & $0.265 \times 10^{-54}$ & 55.3 \\
$6.4545 \times 10^{-14}$ & 325 & 13.0 & $0.479 \times 10^{-54}$ & 100 \\
\hline
\end{tabular}

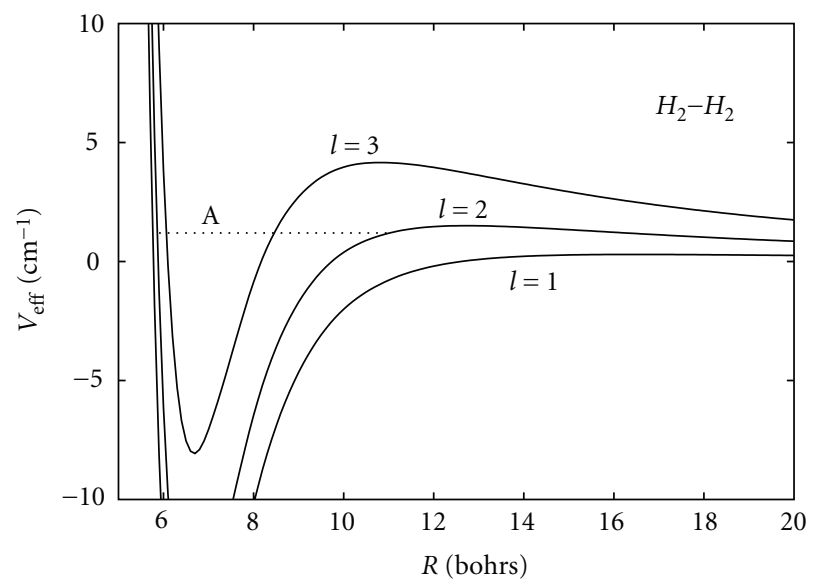

Figure 5: The effective potential for $l=1,2,3$ (isotropic component only). The quasi-bound state or shape resonance energy corresponding to the the feature around $1.2 \mathrm{~cm}^{-1}$ in Figure 4 is indicated with the dotted line.

which is plotted for three values of $l$ in Figure $5 . l$ is the orbital angular momentum corresponding to the classical impact parameter. The reduced mass of the $\mathrm{H}_{2}-\mathrm{H}_{2}$ pair is indicated with $m$ and the distance between the two diatoms with $R$. The anisotropic components $\left(V_{\gamma_{1} \gamma_{2} \gamma}(R)\right.$ with not all indices being zero) of the potential are not shown in Figure 5 but they are included in the calculations of the data shown in Figures 1 through 4.

For the $329 \mathrm{~cm}^{-1}$ frequency shift the $S_{0}(0)$ threshold lies at a collision energy $E=25.4 \mathrm{~cm}^{-1}$. A feature which is due to a predissociating state (B) is clearly discernible about $3 \mathrm{~cm}^{-1}$ below that threshold in Figure 4, consistent with the value for the bound state $\left(j_{1}, j_{2}, l, J\right)=(0,2,0,2)$ which is computed with the same potential in $[26,27]$ Immediately above the threshold two shape resonances $(\mathrm{C}, \mathrm{D})$ corresponding to the final rotational state's effective potential are visible.

To investigate the contribution to the depolarized Raman intensity at $380 \mathrm{~cm}^{-1}$ I have carried out test calculations using different upper limits $E_{\max }$ in the energy integral, (1). The corresponding intensities and their fraction of the total are given in Table 1. It turns out that roughly a fifth of the total intensity at that frequency is contained in the feature which corresponds to the shape resonance labeled with an A in Figures 4 and 5. Furthermore, more than half the intensity comes from energies lower than $25 \mathrm{~cm}^{-1}$ which is close to the $S_{0}(0)$ threshold for $329 \mathrm{~cm}^{-1}$.

\section{Conclusion}

The depolarized Raman spectra at low temperatures has been analysed. Comparison with an experiment carried out at $50 \mathrm{~K}$ [5] verifies satisfactory agreement between the measured and computed spectra. The highly asymmetric $S_{0}(0)$ line profile at $36 \mathrm{~K}$ has been investigated in great detail. The conclusion is that intracollisional interference accounts only for some of the observed asymmetry. The rest stems from the low energy behaviour of the Raman intensity. For example, there is a shape resonance feature which contributes significantly to the intensity for positive detunings $(\nu>$ $\left.354.4 \mathrm{~cm}^{-1}\right)$.

$\mathrm{HD}-\mathrm{He}$ with its very shallow potential well, is less likely to show shape resonance features. This is a possible explanation for that the intra-collisional interference theory and the Fano line shape is so successful in describing the infrared spectrum of $\mathrm{HD}$ in a He bath [20]. Also, since the dipole of HD is so weak, the total intensities of the allowed spectral lines are small. Previous studies of the HD line shapes focused on the regions a few $\mathrm{cm}^{-1}$ from the corresponding transition, compared with $\sim 25 \mathrm{~cm}^{-1} \approx k T$ which are considered here. Thus one expects a smaller role played by threshold effects like that illustrated by comparing the intensities for frequency shifts 329 and $380 \mathrm{~cm}^{-1}$ in Figure 4.

Calculations of low-temperature collision-induced and collision-broadened spectroscopic processes appear to be rather challenging due to resonance features in the cross section. Other methods to handle these are desired and a scheme based on the Breit-Wigner theory [28] similar to that worked out by Bennett et al. [29] for radiative association would likely be useful.

It should also be noted that shape resonances depend strongly on the details of the potential energy surface. This has been investigated in detail for radiative association [30]. The principal aim in this work is to study the mechanisms rather than evaluating accurate light scattering intensity. If high accuracy is needed one should make sure to use the most accurate potential available.

\section{References}

[1] B. J. Berne and R. Pecora, Dynamic Light Scattering, John Wiley \& Sons, New York, NY, USA, 1976.

[2] L. Frommhold, "Collision-induced scattering of light and the diatom polarizabilities," in Advances in Chemical Physics, I. Prigogine and S. Rice, Eds., vol. 46, pp. 1-72, John Wiley \& Sons, New York, NY, USA, 1981.

[3] M. H. Proffitt, J. W. Keto, and L. Frommhold, "Collisioninduced Raman spectra and diatom polarizabilities of the rare gases — an update," Canadian Journal of Physics, vol. 59, no. 10, pp. 1459-1474, 1981.

[4] J. van Kranendonk, "Theory of induced infra-red absorption," Physica, vol. 23, no. 6-10, pp. 825-837, 1957. 
[5] U. Bafile, L. Ulivi, M. Zoppi, F. Barocchi, M. Moraldi, and A. Borysow, "Depolarized-light-scattering spectrum from gaseous hydrogen at $50 \mathrm{~K}$ : the density-squared component," Physical Review A, vol. 42, no. 11, pp. 6916-6919, 1990.

[6] M. Baranger, "General impact theory of pressure broadening," Physical Review, vol. 112, no. 3, pp. 855-865, 1958.

[7] U. Fano, "Pressure broadening as a prototype of relaxation," Physical Review, vol. 131, no. 1, pp. 259-268, 1963.

[8] A. M. Arthurs and A. Dalgarno, "The theory of scattering by a rigid rotator," Proceedings of the Royal Society of London. Series A, vol. 256, p. 540, 1960.

[9] S. Green, "Rotational excitation in $\mathrm{H}_{2}-\mathrm{H}_{2}$ collisions: closecoupling calculations," The Journal of Chemical Physics, vol. 62, pp. 2271-2277, 1975.

[10] M. Gustafsson, L. Frommhold, X. Li, and K. L. C. Hunt, "Roto-translational Raman spectra of pairs of hydrogen molecules from first principles," Journal of Chemical Physics, vol. 130, no. 16, Article ID 164314, 9 pages, 2009.

[11] P. S. Julienne, "Nonadiabatic theory of collision-broadened atomic line profiles," Physical Review A, vol. 26, no. 6, pp. 3299-3317, 1982.

[12] J.-M. Hartmann, C. Boulet, and D. Robert, Collisional Effects on Molecular Spectra, Elsevier, Amsterdam, The Netherlands, 2008.

[13] A. Borysow and M. Moraldi, "Rototranslational Raman scattering in hydrogen," Physical Review A, vol. 40, no. 3, pp. 1251-1261, 1989.

[14] A. P. Kouzov, "Rotational relaxation matrix for fast nonMarkovian collisions," Physical Review A, vol. 60, no. 4, pp. 2931-2939, 1999.

[15] L. Bonamy and J. V. Buldyreva, "Non-Markovian far-wing rotational Raman spectrum from translational modeling," Physical Review A, vol. 63, no. 1, Article ID 012715, 7 pages, 2001.

[16] J. Schäfer and W. E. Köhler, "Low-temperature second virial coefficient of $\mathrm{p}-\mathrm{H}_{2}$ gas obtained from quantum mechanical pair correlation functions," Zeitschrift für Physik D, vol. 13, p. 217, 1989.

[17] X. Li, C. Ahuja, J. F. Harrison, and K. L. C. Hunt, "The collision-induced polarizability of a pair of hydrogen molecules," Journal of Chemical Physics, vol. 126, no. 21, Article ID 214302, 14 pages, 2007.

[18] W. R. Thorson, J. H. Choi, and S. K. Knudson, "Novel theory of the HD dipole moment. II. Computations," Physical Review A, vol. 31, no. 1, pp. 34-42, 1985.

[19] A. R. W. McKellar, "Intensities and the Fano line shape in the infrared spectrum of HD," Canadian Journal of Physics, vol. 51, no. 4, pp. 389-397, 1973.

[20] A. R. W. McKellar and N. H. Rich, "Interference effects in the spectrum of HD: II. The fundamental band for HD-rare gas mixtures," Canadian Journal of Physics, vol. 62, no. 12, pp. 1665-1672, 1984.

[21] P. G. Drakopoulos and G. C. Tabisz, "Far-infrared rotational spectrum of HD: line shape, dipole moment, and collisional interference," Physical Review A, vol. 36, no. 12, pp. 5556$5565,1987$.

[22] R. M. Herman and J. C. Lewis, "Scalar collisional interference parameters for the $\mathrm{HD}_{1}(0)$ and $\mathrm{R}_{1}(1)$ lines in mixtures with He," in Spectral Line Shapes 11, J. Seidel, Ed., vol. 559 of AIP Conference Proceedings, pp. 397-399, The American Institute of Physics, New York, NY, USA, 2000.
[23] M. Gustafsson and L. Frommhold, "The HD-He complex: interaction-induced dipole surface and infrared absorption spectra," Journal of Chemical Physics, vol. 115, no. 12, pp. 5427-5432, 2001.

[24] M. Gustafsson and L. Frommhold, "Intracollisional interference of R lines of HD in mixtures of deuterium hydride and helium gas," Physical Review A, vol. 63, no. 5, Article ID 052514, 6 pages, 2001.

[25] A. Borysow and M. Moraldi, "Effects of the intermolecular interaction on the depolarized rototranslational Raman spectra of hydrogen," Physical Review A, vol. 48, no. 4, pp. 30363046, 1993.

[26] J. Schäfer and A. R. W. McKellar, "Faint features of the rotational $\mathrm{S}_{0}(0)$ and $\mathrm{S}_{0}(1)$ transitions of $\mathrm{H}_{2}$ : a comparison of calculations and measurements at $77 \mathrm{~K}$," Zeitschrift für Physik $D$, vol. 15, p. 51, 1990.

[27] J. Schäfer and A. R. W. McKellar, "Erratum: Faint features of the rotational $S_{0}(0)$ and $S_{0}(1)$ transitions of $\mathrm{H}_{2}$," Zeitschrift für Physik D, vol. 17, no. 3, p. 231, 1990.

[28] G. Breit and E. Wigner, "Capture of slow neutrons," Physical Review, vol. 49, no. 7, pp. 519-531, 1936.

[29] O. J. Bennett, A. S. Dickinson, T. Leininger, and F. X. Gada, "Radiative association in $\mathrm{Li}+\mathrm{H}$ revisited: the role of quasibound states," Monthly Notices of the Royal Astronomical Society, vol. 341, no. 1, pp. 361-368, 2003.

[30] M. Jurek, V. Spirko, and W. P. Kraemer, "Ab initio determination of the rate coefficient for radiative association of $\mathrm{He}\left({ }^{1} \mathrm{~S}\right)$ + $\mathrm{H}^{+}$," Chemical Physics, vol. 193, no. 3, pp. 287-296, 1995. 


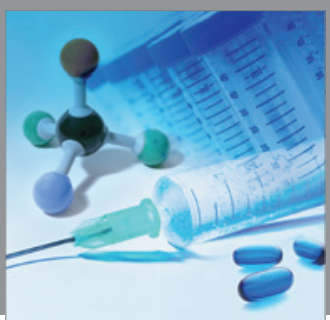

International Journal of

Medicinal Chemistry

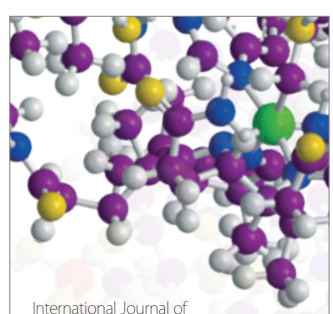

Carbohydrate Chemistry

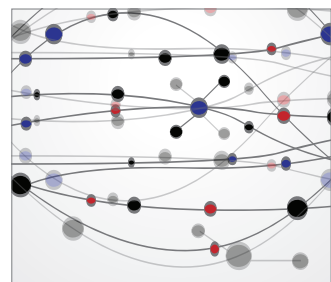

The Scientific World Journal
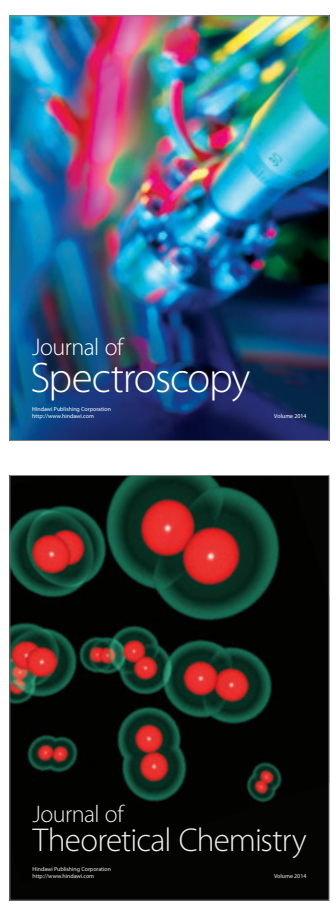
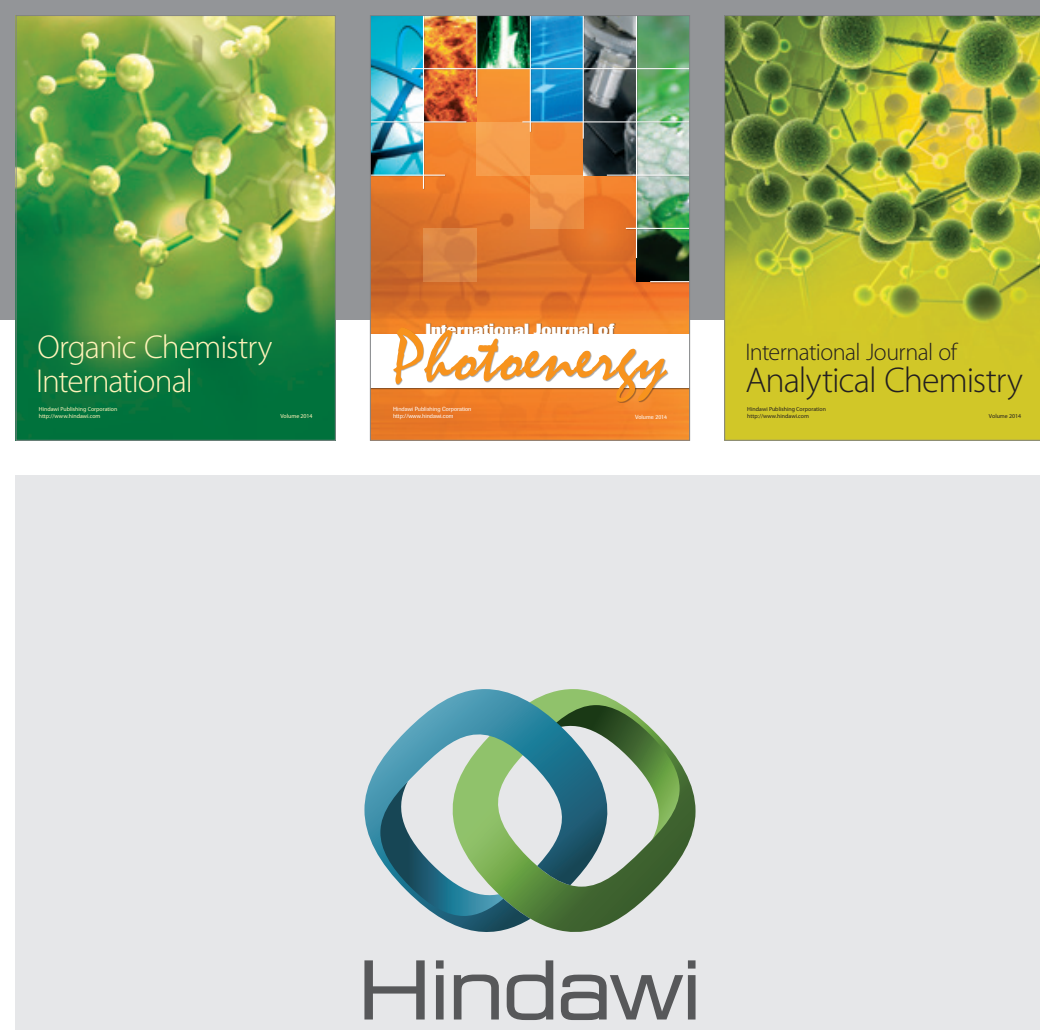

Submit your manuscripts at

http://www.hindawi.com
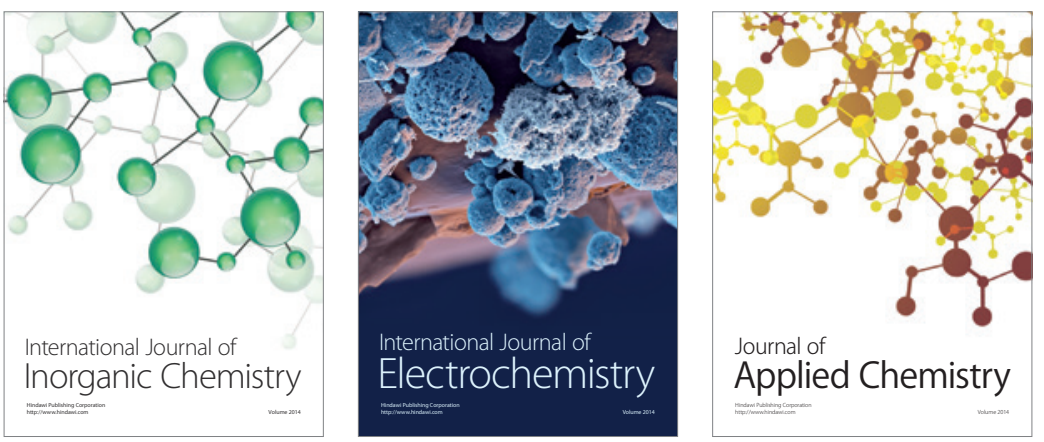

Journal of

Applied Chemistry
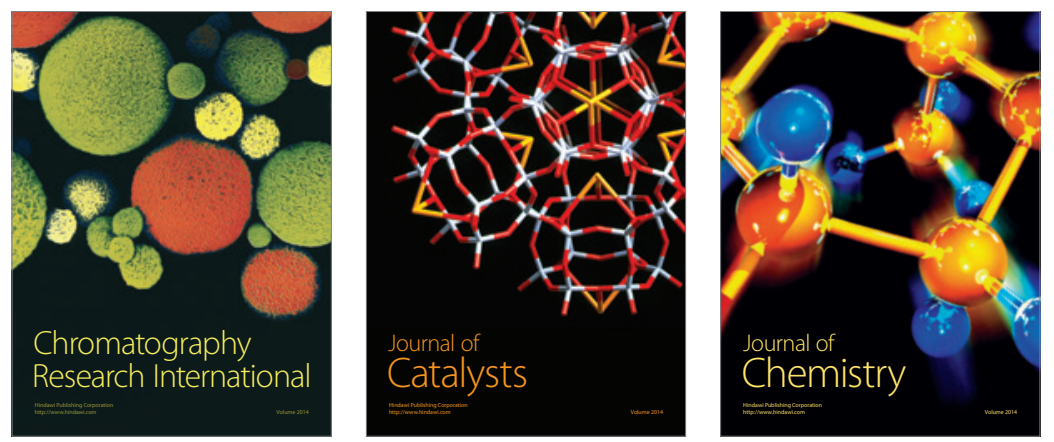
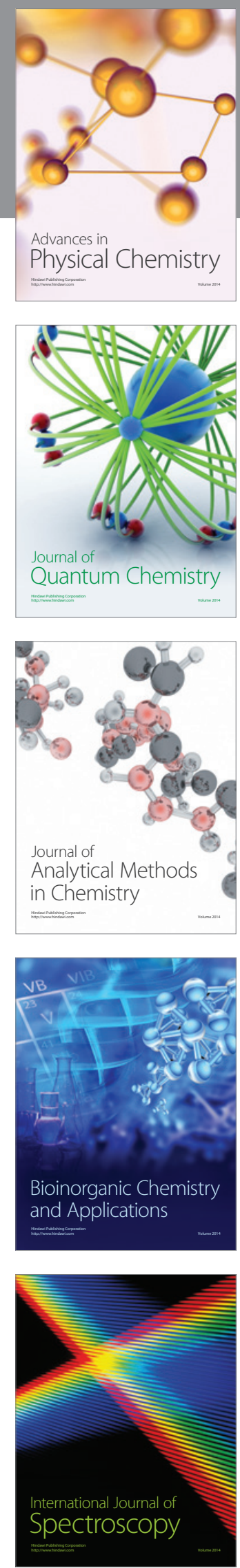